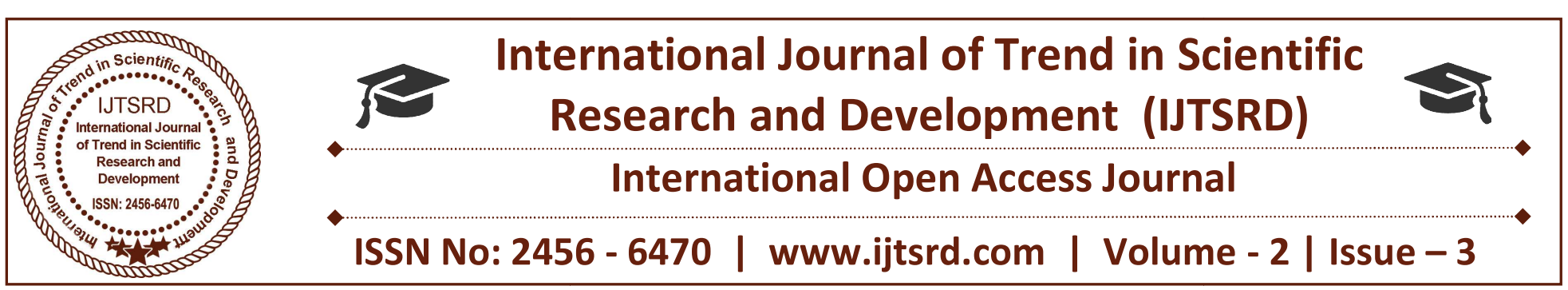

\title{
Secure Authentication for Advanced Voting System using Fingerprint and RFID
}

\author{
B. Shalini \\ Department of Computer Science and Engineering, \\ GKM College of Engineering and Technology, \\ Chennai, Tamil Nadu, India
}

\author{
M. Sakthi Prabha \\ Department of Computer Science and Engineering, \\ GKM College of Engineering and Technology, \\ Chennai, Tamil Nadu, India
}

\section{R. Krithika}

Assistant Professor, Department of Computer Science and Engineering, GKM College of Engineering and Technology, Chennai, Tamil Nadu, India

\begin{abstract}
The problem of voting is still critical in terms of rigging and security. $\mathrm{A}$ RFID and BIOMETRIC fingerprint sensor is being used in this advanced voting system. In the initial stage of voting we use bullet papers and polling machine without any unique authentication on the election voting process. Biometric are used for human secure authentication because fingerprint are unique in human biological nature. RFID (Radio Frequency Identification) used for automatic fetching data from data stored in device. The both modules are enhanced the rigging security in the election.
\end{abstract}

Keywords: RFID tag and reader, fingerprint sensor, microcontroller PIC16F877A.

\section{INTRODUCTION}

This paper focuses on problem faced by the election commission in rigging vote during the election. To overcome this problem, we introduced the bio-metric fingerprint verification for the voting in the election. (fingerprint is unique in nature for each person even in the case of twins) RFID tags have the candidate details. Radio frequency identification technology allows time to be scanned quickly and enable fast identification of the particular. Even when it is covered by several items. It enables get the candidate profile faster. Both of the fingerprint verification and the RFID technology makes candidate cast their vote secure and authenticate.

\section{RELATED WORK}

Robust Biometric Recognition from Palm Depth Images for Gloved Hands, P. Nguyen, Member, 2015disadvantage is Biometric recognition can be used to improve gesture-based interfaces by automatically identifying operators. Low computational cost multilayer graph-based segmentation algorithms for hand recognition on mobile phones, Danil de Santos-sierra,2014 disadvantages is Unconstrained and contact-free hand recognition problem with mobile devices is not solved yet because these systems have to deal with hard problems like different backgrounds and illumination. Palm-Print Classification by Global Features, Bob Zhang, 2013 Three-dimensional palm prints are harder to counterfeit than 2-D palm prints and more robust to variations in illumination and serious scrabbling on the palm surface. Previous work on 3-D palm-print recognition has concentrated on local features such as texture and lines.

\section{PROPOSED SYSTEM}

The Biometric voting system with RFID and biometric fingerprint using voter id. Only eligible voters are permitted to cast their vote. Removes human fallibility from the tabulation process and makes your election results available within limited period of time at the end of the election. No paper ballots, no postage and no printing It will be secured and there will be no fake votes. 
The following process involved in our proposed system:

RFID and Bio-Metric Finger Print Sensor is the first stage of the system, where the person place the RFID tag in the reader. The reader checks the matching data and moves to next stage. .

- Fingerprint is taken and checked, with the existing database of the area. This unit works as your voter id where they identify, whether the person resident of that area or not.

- Any proxy person try to vote and his data was not there means the red light will glow and he is not allowed to enter the voting stage and it also blocks the person who comes to vote twice.

- Voting Area is the second stage of the system, where the person who will go to push the button of his choice And the count is stored in microcontroller and send to counting station via RF immediately.

- Third stage is the Counting Area stage. In this stage information received from the voting station will be added and displayed parallel on the display, this will happen in real time.

- By using biometric fingerprint it provides enough security which reduces the false votes.

\section{PIC 16F877A CONTROLLER}

The $16 \mathrm{~F} 877 \mathrm{~A}$ is a capable microcontroller that can do many tasks because it has a large enough programming memory (large in terms of sensor and control projects) 8k words and 368 Bytes of RAM. This is enough to do many different projects.

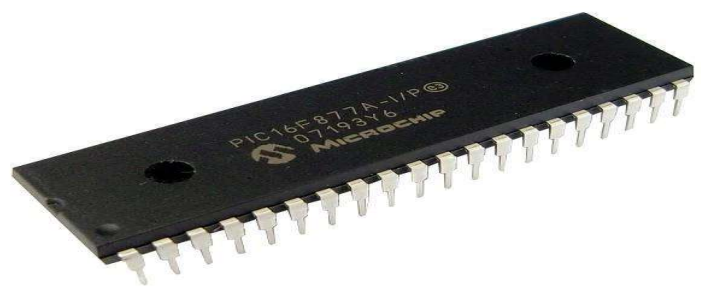

PIC 16F877A CONTROLLER

\section{BLOCK DIAGRAM}

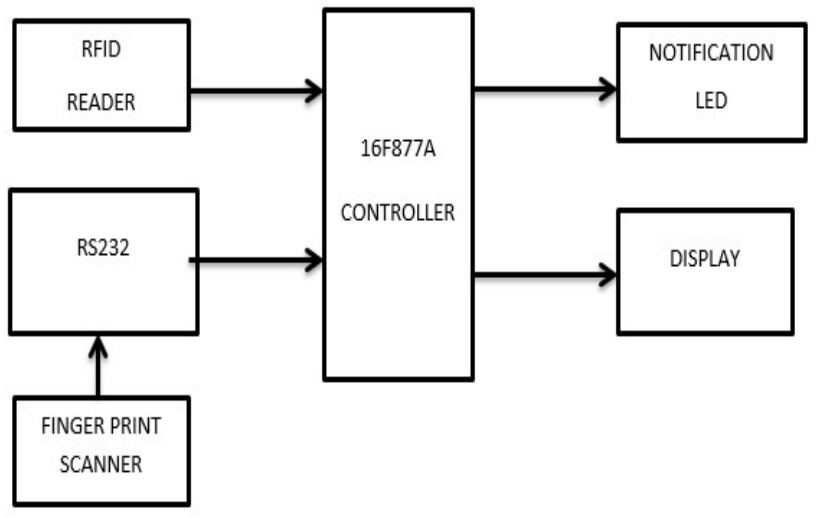

\section{SYSTEM HARDWARE}

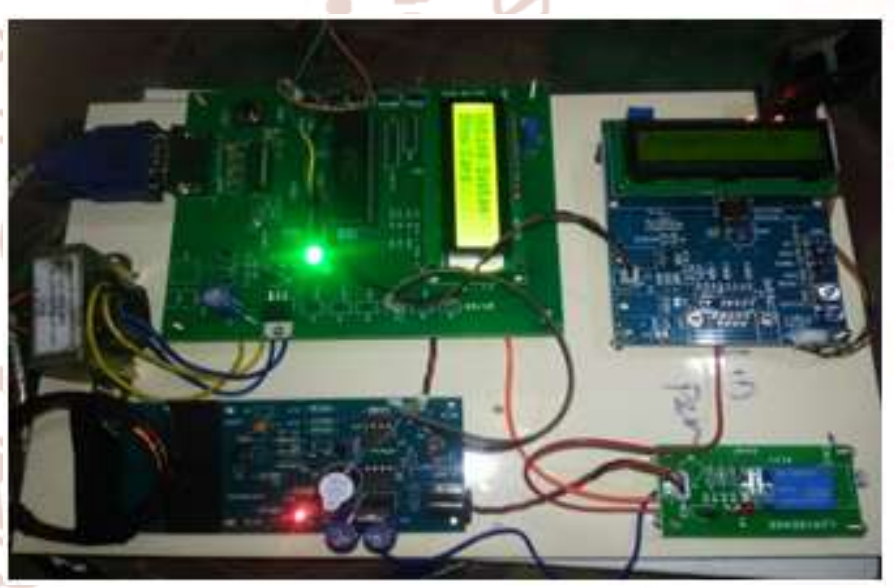

Fig 1: PROPOSED SYSTEM 
ARCHITECTURE DIAGRAM

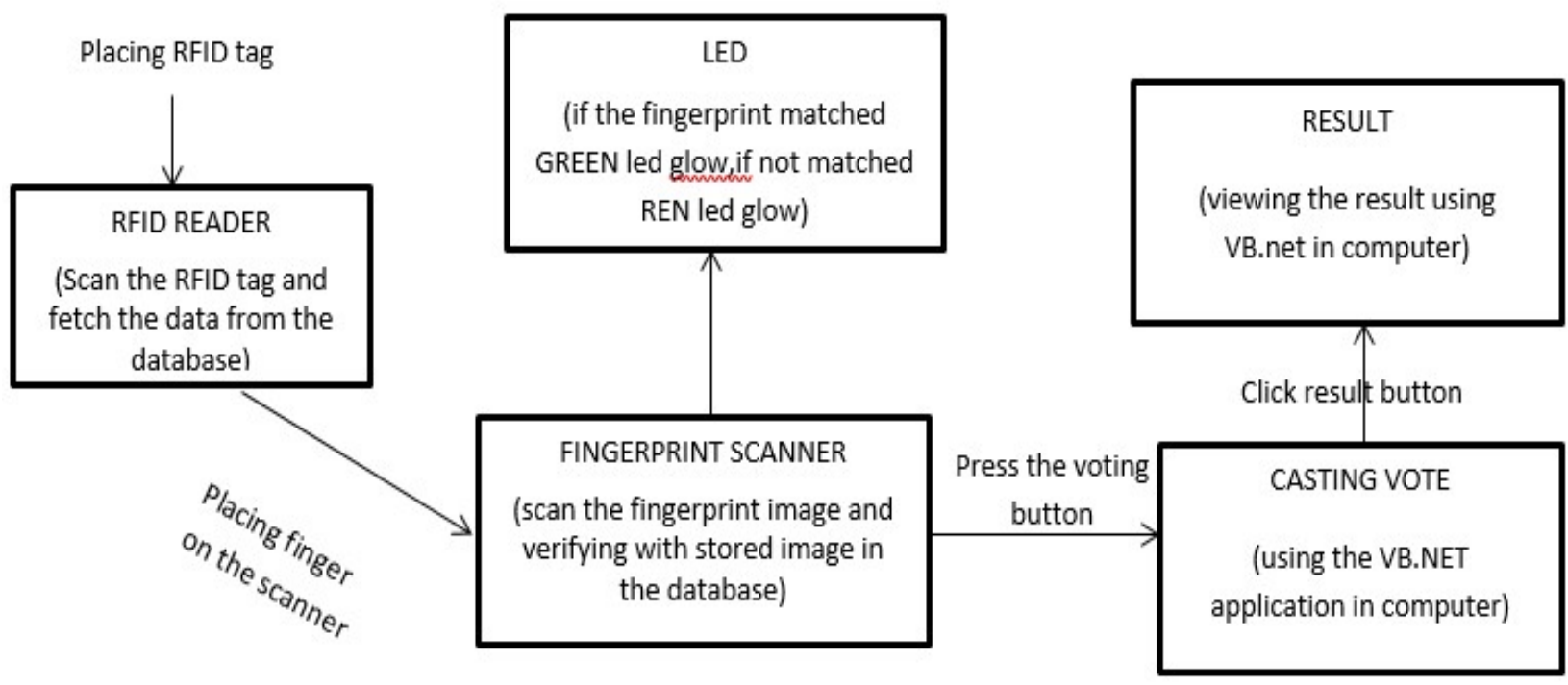

Fig 2: Working Architecture

\section{LIST OF WORKING MODULES IN PROPOSED SYSTEM}
a) RFID module
b) Fingerprint verification module
c) Casting module
d) Counting module

\section{a) RFID MODULE}

In this module we are implement RFID tags and reader, RFID contain voters ID details and RFID reader is used to scan the tag and fetch the voter's details from the database. Radio frequency identification technology allows time to be scanned quickly and enable fast identification of the particular

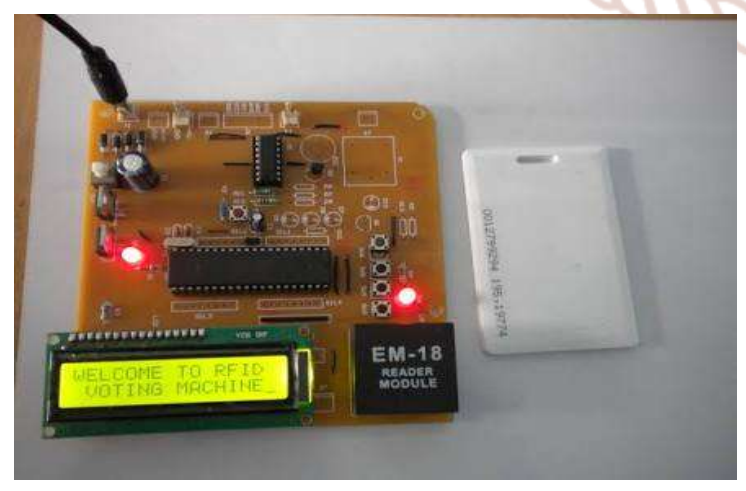

\section{c) CASTING MODULE}

This module is used to identify the specific constitution volume of voter and also viewing the list of candidates those who are nominated for particular volume in particular position. This module will help the voters they can easily identify the candidates in that system.

Fig 3: RFID Tag \& Reader 


\section{CASTING VOTE}

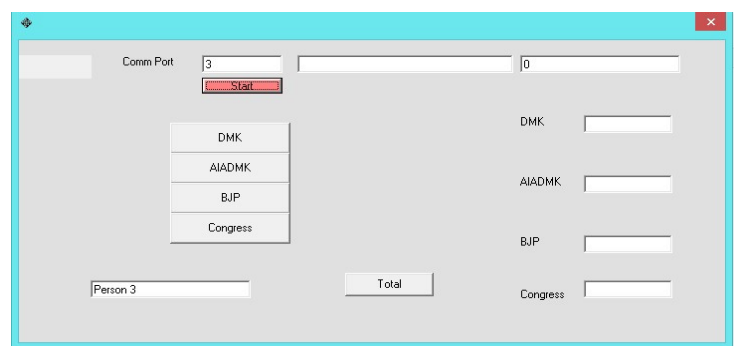

Fig-5: Before Casting

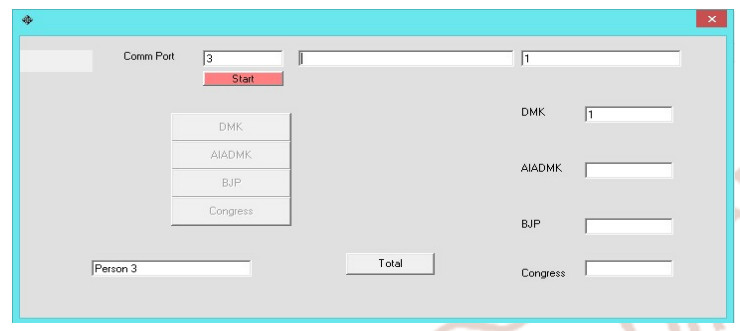

Fig-6: After Casting

\section{d) COUNTING MODULE}

This module will count the number of votes received from user applications to Database once they processed the action, voter should be revoked they are not allowed to perform further voting process in a specific duration.

\section{Result Window}

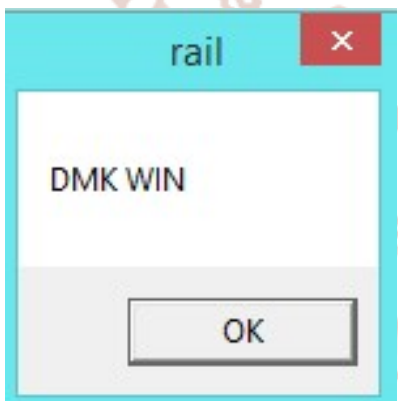

\section{CONCLUSION}

After implementation of this project we can change the face of today's voting system making it more secure and corruption less. It will give a fair chance to every leader to win on the basis of strength of money and power. The scope of the project can be raised to the society, institutional or nation level by using a more secure and efficient database management system that could handle hundreds, thousands or billions of users.in future we implement iris authentication for security.

\section{REFERENCE}

1. Burmester, M., Magkos, E., Towards secure and practical e-elections in the new era. In D. Gritzalis, editor, Secure Electronic Voting, pages 63-72. Kluwer Academic Publishers, (2003).

2. ARM Cortex M3\|, [Online] Available: http://www.st.com

3. A. illafiorita and K. Weldemariam, and. iella, "Development, Formal erification, and Evaluation of an E-voting System with PA," IEEE transactions on Information Forensics and Security, vol. 4, no. 4, 2009

4. Qadah, G.Z., Taha, R.: Electronic voting systems: requirements, design, and implementation. Compute. Stand. Interf. 29(3), 376-386 (2007).

5. A. M. Keller, A. Dechert, K. Auerbach, D. Mertz, A. Pearl, and J. L. Hall, "A PC-based OpenSource Voting Machine with an Accessible VoterVerifiable Paper Ballot," Proceedings of the USENIX Annual Technical Conference, U.S.A., 2005, p.52. 